\title{
Determination of point estimates in an information- measuring system on the basis of light shields
}

\author{
V. A. Aphanasiev', S. I. Yuran² \\ ${ }^{1}$ Kalashnikov Izhevsk State Technical University, Izhevsk, Russia \\ ${ }^{2}$ Izhevsk State Agricultural Academy, Izhevsk, Russia \\ ${ }^{1}$ Corresponding author \\ E-mail: 1double_ava@mail.ru, ${ }^{2} y u r a n-49 @ y a n d e x . r u$
}

Received 10 December 2018; received in revised form 20 February 2019; accepted 27 March 2019 DOI https://doi.org/10.21595/jme.2019.20442

Check for updates

Copyright (C) 2019 V. A. Aphanasiev, et al. This is an open access article distributed under the Creative Commons Attribution License, which permits unrestricted use, distribution, and reproduction in any medium, provided the original work is properly cited.

\begin{abstract}
In the article, we research nonlinear estimations of a mathematical expectation of a random value received by the criterion of the minimum amount of deflections in a production degree that determinates a deviation weight. The research results can be used for the estimation of a mathematical expectation and determination of effectiveness of estimating in an information-measuring system based on light shields.
\end{abstract}

Keywords: point estimate, average value, standard deviation, least square method, the method of least modules.

\section{Introduction}

In different information-measuring systems, including an information-measuring system based on light shields $[1,2]$ characteristics of random values according to independent samples are important. One of these characteristics is a mathematical expectation. In this case, evaluations for point and interval estimation of for the control of statistical hypotheses are searched [3-5]. Especially in a case of a point and interval estimation the criterion of valuation optimality (statistics $y\left(x_{1}, \ldots\right.$, $x_{2}$ ) - the function of sample values $x_{1}, \ldots, x_{n}$ that does not depend on sample volume $n$ ) is minimum of its dispersion $\sigma^{2}$. An effective valuation has a minimum amount of dispersion. In the case of a statistical control of hypotheses, the criterion of the optimality is taken the minimum of a total risk (the sums of error probabilities of I kind $\alpha$ and II kind $\beta$ ), or minimum $\beta$ at a fixed $\alpha$. It is obvious that the smaller a total risk is, the sharper an integral function of evaluation distribution $F(y)$ is $[5,6]$.

\section{The estimation of a mathematical expectation}

Most common random values is distributed to a normal law $N\left(\mu, \sigma^{2}\right)$ with a mathematical expectation $\mu$ and dispersion $\sigma^{2}$. A wide distribution of a normal law is proved by a central limit theorem of probability theory $[4,5]$. Moreover, it follows from the uncertainty principle (entropy according to K. Shennon) in the case of stable test conditions [7]. In the case of a normal random value, its distribution is symmetrical concerning a mathematical expectation, for the valuation of a mathematical expectation we take random average value:

$\bar{x}=\frac{1}{n} \sum_{i=1}^{n} x_{i}$

Median $\bar{x}_{M e}$ average values $r$ - statistics $\bar{r}_{1, n}=\frac{1}{n}\left(x_{(1)}+x_{(n)}\right), \bar{r}_{2, n-2}=\frac{1}{n}\left(x_{(2)}+x_{(n-1)}\right)$ and so on, where $x_{(r)}-r$-statistics [8].

According to a famous theorem of A. A. Markov [5] an effective (with minimum dispersion) linear estimation (a linear function from random values) of a mathematical expectation at any law of distribution in the case of an independent sampling with equidistant changes is a random average 
value $\bar{x}$ Eq. (1). The dispersion of valuation $\bar{x}$ is inversely proportional to the sample volume and in the case of small samples $(n<10, \ldots, 20)[9,10]$ the problem of the search of more effective valuations in the area of nonlinear functions is actual.

The valuation Eq. (1) follows from the criterion of the optimality according to the least square method (LSM):

$Q_{2}=\sum_{i=1}^{n}\left(x_{i}-\theta\right)^{2}=\min$

A necessary condition of the extremum of the function Eq. (2) at $\theta$, gives a linear valuation Eq. (1). In the case of the criterion of a general view:

$Q_{v}=\sum\left|x_{i}-\theta_{v}\right|^{v}=\min$

If $v \neq 2$ we get nonlinear valuations, the valuation of least module method (LMM) is a frequent case if $v=1[8]$.

If $v>1$, then in the valuation the weights of big deflection are increased, if $v<1$, then vice versa. So, to take the valuations with $v>2$ is objectionable. From the point of view of reducing the influence of abnormal measurement, it is appropriate to take the valuations with $v<1$. For defining of valuations on the criterion of a general view the method of variationally weighted quadratic approximation is developed. It can be used only if $v<2$. If $v<1$, some problems can appear than can be linked to the presence $n-1$ local (local extremum) function Eq. (3) and dependence of the result of the decision from an initial estimate.

\subsection{Determination of the estimation of a mathematical expectation according to the method of least modules}

Now we demonstrate, that in the case $\operatorname{MLM}(v=1)$ the valuation $\theta_{1}$ on the criterion Eq. (3) can be found without using the method of variationally weighted quadratic approximation in a trivial way. $\theta_{1}$ is the valuation of a mathematical expectation in the case of the method Eq. (3) if $v=1$. We consider the sums of the deflection modules for two extreme $r$-statistics $x_{(1)}, x_{(n)}$. The dependence of this sum on $\theta_{1}$ has the form, shown in the Fig. 1.

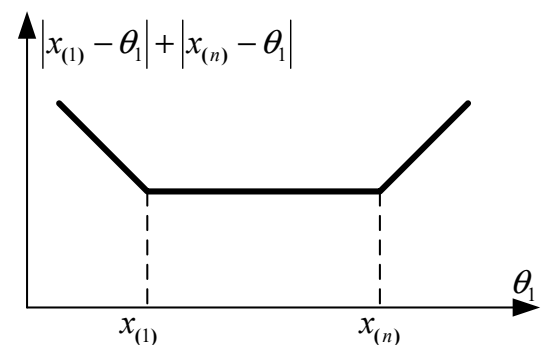

Fig. 1. The dependence of the sum of the deflection modules on $\theta_{1}$

The dependence of the sum of the deflection modules of the following $r$-statistics $x_{(2)}, x_{(n-1)}$, put in the interval $\left(x_{(1)}, x_{(n)}\right)$, has a similar form and the minimum value of a total sum will be within the interval $x_{(2)}, x_{(n-1)}$ and so on. Thus, the minimum value of the sum will be when $\theta_{1}=x_{\frac{n+1}{2}}$, if $n$-odd, or in the interval $\left(x_{\left(\frac{n}{2}\right)}+x_{\left(\frac{n+2}{2}\right)}\right)$ if $n$ - even. In this case an optimal value can be taken equal to the middle of the interval $\theta_{1}=\frac{1}{2}\left(x_{\left(\frac{n}{2}\right)}+x_{\left(\frac{n+2}{2}\right)}\right)$. Therefore, MLM-valuation coincides with the median value $\bar{x}_{M e}$. From this, it follows that MLM-valuation is an unbiased estimate of a 
mathematical expectation for symmetric distributions. It concerns other valuations $\theta_{v}$ if $v \neq 2$ as well.

Despite the fact that the efficiency of values for $v \neq 2$ is smaller, the efficiency of an average value $\theta=\frac{1}{2}\left(\theta_{2}+\theta_{v}\right)$ can be close to the efficiency of the value $\theta_{2}=\bar{x}$ thanks to the thing that the correlation coefficient between $\theta_{2}$ and $\theta_{v}$ is not equal to 1 . If $v \leq 1$, then an average value is less sensitive to abnormal change. In the case if $v=1$ the correlation coefficient (when $n$ is odd) between $\theta_{2}=\bar{x}$ by Eq. (1) and $x_{\left(\frac{n+1}{2}\right)}$ is easy to calculate. It is equal to $\rho=-\frac{1}{n-1}, n \geq 2$ [8].

We also notice that, in the case of hypothesis testing about a mathematical expectation its value for the main hypothesis is known. It removes the problem of the choice of an initial estimate at a finding of an optimal value $\theta_{v}$ Eq. (3). The value:

$\theta_{o p}=\frac{1}{2}\left(\theta_{1}+\theta_{2}\right)=\frac{1}{2 n} \sum_{r}^{n} x_{(r)}+\frac{1}{2} x_{\left(\frac{n+1}{2}\right)}$.

Leads onto the idea to build a linear value of this kind:

$\theta=\sum_{r=1}^{n} c_{r} x_{r}$

where $r$-statistics are taken with weight coefficients $c_{r}$.

Coefficients $c_{r}$ can be calculated, using the MLS-criterion, for example, taking into consideration an additional condition - the immutability of the value. So, the decision brings to the task on a conditional extremum of the function. However, for the decision it is necessary to know the correlation coefficients between $r$-statistics and their dispersion.

\subsection{Criterions of the optimality of the valuation of a mathematical expectation}

So now, we consider two more approaches. In the first case we will use the criterion:

$\sum_{r=1}^{n}\left(x_{(r)}-\mu-\sigma \mu_{r}\right)^{2}=\min$,

where $\mu-$ a mathematical expectation of a random value, $\mu_{r}-$ a mathematical expectation $r$ - statistics of a random value according to the law $N(0,1)$. The necessary condition of the extremum for Eq. (6) (according to MLS) gives the values:

$\mu=\bar{x}=\frac{1}{n} \sum_{r=1}^{n} x_{(r)}=\frac{1}{n} \sum_{i=1}^{n} x_{i}$

$\sigma=s=\frac{\sum x_{(r)} \mu_{r}}{\sum\left(\mu_{r}\right)^{2}}$

To get values the ratio is used $\sum_{r=1}^{n} \mu_{r}=0$ in the case of a symmetric distribution.

In the second case we take the criterion of the minimum of sum of squared deviations $r$-statistics $x_{(r)}$ from calculated - quantiles of a normal law $N\left(\mu, \sigma^{2}\right)$, corresponding probability:

$p_{r}=\frac{2 r-1}{2 n}, \quad \sum_{r=1}^{n}\left(x_{(r)}-F_{N}^{-1}\left(p_{r}, \mu, \sigma\right)\right)^{2}=\min$,

$\sum_{r=1}^{n}\left(p_{(r)}-F_{N}\left(x_{(r)}, \mu, \sigma\right)\right)^{2}=\min$,

where $F_{N}$ and $F_{N}^{-1}$ - an integral function of distribution and its inverse function for a normal law 
$N\left(\mu, \sigma^{2}\right)$.

\subsection{Determination of effectiveness and unbiasedness of the valuation of a mathematical expectation}

For the determination of effectiveness and unbiasedness of the valuation of a mathematical expectation a statistical modeling with the help of the software package MathCAD was taken [11-13]. The algorithm of the modeling was following.

1. Independent ones $j=1, \ldots, N$, the sample volume $i=1, \ldots, n$, each from the law $N(0,1)$ were modeled.

2. For each sample valuations $y_{0 j}=\theta_{2 j}, y_{1 j}=\theta_{1 j}, y_{2 j}=\theta_{\frac{1}{6} j}$ were found. Two final valuations were found with the help of the function Minerr for Eq. (3) at zero value of an initial approximation.

3. According to given values, average values $y_{3 j}=\frac{1}{2}\left(y_{0 j}+y_{1 j}\right), y_{4 j}=\frac{1}{2}\left(y_{0 j}+y_{2 j}\right)$ were found.

4. For each value sample mean value:

$\bar{y}=\frac{1}{N} \sum_{j=1}^{N} y_{j}$

Sample dispersion:

$D_{y}=\frac{1}{N-1} \sum_{j=1}^{N}\left(y_{j}-\bar{y}\right)^{2}$

and mean-square sample deviation were received:

$\sigma_{x}=\sqrt{D_{y}}$

5. Coefficients of correlation between values $y_{0}, y_{1}$ and $y_{0}, y_{2}$ were determined:

$\rho_{0 i}=\frac{1}{\sigma_{y 0} \sigma_{y i}(N-1)} \sum_{j=1}^{N}\left(y_{0 j}-\bar{y}_{0}\right)\left(y_{i j}-\bar{y}_{i}\right)$.

6. With the help of the sort function $y y=\operatorname{sort}(y)$ the sets of $r$ - statistics of valuation were formed and according to them empirical integral functions of distribution as dependencies $j / N$ on $y y_{j}$, where $j=1, \ldots, N$ were built.

7. The effectiveness of values $e_{y i}=\frac{\sigma_{y i}}{\sigma_{y 0}}$, where $i=1, \ldots, 4$ compared to the value $y_{0}=\bar{x}$ at the sample average was determined.

The results of calculations are demonstrated in the Table 1 (number of samples $N=10000$ ).

Table 1. Effectiveness and coefficients of correlation of values $y i$

\begin{tabular}{|c|c|c|c|c|c|c|c|}
\hline$n$ & $\sigma_{y 0}$ & $e_{y 1}$ & $e_{y 2}$ & $e_{y 3}$ & $e_{y 4}$ & $\rho_{01}$ & $\rho_{02}$ \\
\hline 4 & 0,500 & 0,839 & 0,964 & 0,939 & 0,839 & $-0,539$ & $-0,635$ \\
\hline 8 & 0,354 & 0,811 & 0,952 & 0,817 & 0,817 & $-0,546$ & $-0,656$ \\
\hline 12 & 0,285 & 0,789 & 0,932 & 0,794 & 0,794 & $-0,552$ & $-0,669$ \\
\hline 16 & 0,249 & 0,800 & 0,934 & 0,786 & 0,786 & $-0,556$ & $-0,670$ \\
\hline 20 & 0,223 & 0,778 & 0,931 & 0,778 & 0,778 & $-0,557$ & $-0,677$ \\
\hline
\end{tabular}

In the next numeral experiment $(N=100000)$ the values of a mathematical expectation under 
$r-$ statistics $z_{1}=x_{\left(\frac{n+1}{2}\right)}, z_{2}=\frac{1}{2}\left(x_{\left(\frac{n-1}{2}\right)}+x_{\left(\frac{n+3}{2}\right)}\right)$ and $z_{3}=\frac{1}{3}\left(x_{\left(\frac{n-1}{2}\right)}+x_{\left(\frac{n+1}{2}\right)}+x_{\left(\frac{n+3}{2}\right)}\right)$ $\left(n-\right.$ an odd number) were investigated. In addition, average values $z z i=1 / 2\left(z_{1}+z_{i}\right)$ were found as well.

Analogically, the general effectiveness was compared with the effectiveness of the valuation at the sample mean $y_{0}=\bar{x}$. For this connections $e_{z i}=\sigma_{z i} / \sigma_{y 0}$ and $e_{z z i}=\sigma_{z z i} / \sigma_{y 0}$ were calculated. Moreover, coefficients of correlation of values $z i$ with $y_{0}$ were calculated. The results of calculations are demonstrated in the Table 2.

Table 2. Effectiveness and coefficients of correlation of values $z i$ with $y 0$

\begin{tabular}{|c|c|c|c|c|c|c|c|c|c|c|}
\hline$n$ & $\sigma_{y 0}$ & $e_{z 1}$ & $e_{z 2}$ & $e_{z 3}$ & $e_{z z 1}$ & $e_{z z 2}$ & $e_{z z 3}$ & $\rho_{z 1, y 0}$ & $\rho_{z 2, y 0}$ & $\rho_{z 3, y 0}$ \\
\hline 5 & 0,448 & 1,194 & 1,074 & 1,064 & 1,051 & 1,019 & 1,019 & 0,834 & 0,931 & 0,938 \\
\hline 9 & 0,333 & 1,224 & 1,131 & 1,134 & 1,061 & 1,035 & 1,036 & 0,819 & 0,886 & 0,884 \\
\hline 13 & 0,278 & 1,227 & 1,154 & 1,159 & 1,060 & 1,040 & 1,041 & 0,811 & 0,862 & 0,859 \\
\hline 17 & 0,243 & 1,234 & 1,176 & 1,180 & 1,063 & 1,047 & 1.048 & 0,810 & 0,850 & 0,847 \\
\hline 21 & 0,219 & 1,237 & 1,188 & 1,192 & 1,064 & 1,049 & 1,051 & 0,806 & 0,840 & 0,837 \\
\hline
\end{tabular}

\section{Results}

On the basis of the analysis of modelling results the following conclusions can be done.

1. Accurate evaluation $z_{1}$ according to MLM is less effective compared to $y_{0}$ at the sample mean $\left(e_{z 1}>1\right.$, Table 2$)$. At the same time the approximate evaluation $y_{1}$ according to MLM, received with the help of the function Minner at an initial estimate, equal to a mathematical expectation is more effective $\left(e_{y 1}<1\right.$, Table 1). If in the case of an initial estimate to take the sample mean $y_{0}=\bar{x}$, then the effectiveness decreases to about value $e_{z 1}$. As a result, approximate evaluations are more effective, and they can be used in the case of checking of statistical hypotheses when a mathematical expectation is supposed known (hypothetically)

2. Accurate evaluations at two or three average members of the sample ( $r$ - statistics) according to the effectiveness approach the sample mean (Table 2). Their advantage is insensitivity to abnormal changes.

3. The estimate of standard deviation (e.s.d.) by Eq. (8) is unbiased and by efficiency, it coincides with the value by sample e.s.d.

4. The estimation of a mathematical expectation by Eqs. (9), (10) are unbiased, but by efficiency they concede the value by the sample mean.

5. Values of e.s.d. $\sigma$ by Eqs. (9), (10) are a bit low, the value by Eq. (9) is more accurate. By efficiency the valuations concede the value by the sample mean of e.s.d.

6. An empirical integral function of distribution of average value $y_{3}$ is sharper than functions of distribution of values $y_{0}=\bar{x} n y 1$. Empirical integral functions of distribution of average point estimates $z z_{2}, z z_{3}$ practically coincide with an integral function of distribution of the value $y_{0}=\bar{x}$.

7. Since the values of coefficients of correlation in the Table 1 are not equal to \pm 1 (Table 1 ), then between $y_{0}$ and $y_{1}$, as well as between $y_{0}$ and $y_{2}$ the dependence is nonlinear.

8. In terms of estimation values of coefficients of correlation in the Table 2, we can conclude that dependences between values $y_{0}$ and $z_{1}, z_{2}, z_{3}$ are more linear.

Also, it is necessary to mention that for a normal random value we were not able to find a superefficient nonlinear value [7]. The expediency of using concrete values depends on a situation that is indicated in the work above.

\section{Conclusions}

The use of estimates, received in the research process, let raise the accuracy of determination of point estimates when a bullet hits the target in information-measuring systems based on light 
automatic shields that are intended for small arms testing.

The approximate evaluation of the sample mean $y_{1}$ is more effective than the accurate evaluation $z_{1}$ and can be used in the case of statistical hypotheses testing when a mathematical expectation is hypothetically known. If one uses the approximate evaluation of the sample mean, it leads to the rise of the accuracy of determination of point estimates when a bullet hits the target in 1,5-2 times. This conclusion is experimentally confirmed in the research [1].

The accurate evaluations of the sample mean by two or more means of the sample are insensible to abnormal measurements and can be used in small arms testing in the conditions of different noises.

\section{References}

[1] Aphanasiev V. A., Kazakov V. S., Korobeinikov V. V. An experimental research of the influence of operating threshold in a light target. Journal of Intellectual Systems in Manufacture, Vol. 21, Issue 1, 2013, p. 116-119.

[2] Aphanasiev V. A., Lyalin V. E. An experimental research of the effectiveness of using of weighted time points in a light target. Works of International Symposium Reliability and Quality, Vol. 1, 2013, p. 245-248.

[3] Wentzel E. S. Theory of Probabilities. High School, Moscow, 2001, p. 480.

[4] Ivchenko G. I., Medvedev U. I. Mathematical Statistics. High School, Moscow, 1984, p. 248.

[5] Wilks S. Mathematical Statistics. Science, Moscow, 1967, p. 632.

[6] Gaskarov D. V., Shapovalov V. I. Small Sample. Statistics, Moscow, 1978, p. 248.

[7] Mudrov V. I., Kushko V. A. Methods of Change Processing. Sov. Radio, Moscow, 1976, p. 192.

[8] David G. Ordinal Statistics. Science, Moscow, 1979, p. 336.

[9] Aphanasieva N. U. Numeral and Experimental Methods of a Scientific Experiment. Publishing KnoRus, Moscow, 2009, p. 276.

[10] Aphanasieva N. U., Aphanasiev V. A., Verkienko U. V. Theory of Probability and Mathematical Statistics: an Educational Material for Students of the Speciality 230101 "Computing Machines, Complexes, Systems and Networks". Publishing IzSTU, Izhevsk, 2006, p. 246.

[11] Diyakonov V. N. Mathcad 11/12/13 in Maths. Guidebook. Hotline, Telekom, Moscow, 2007, p. 958.

[12] Diyakonov V. N., Abramenkova I. V. Mathcad 7 in Maths, Physics and the Internet. Publishing Knowledge, Moscow, 1998, p. 352.

[13] Financial, Engineering and Scientific Calculations in Area Windows 95. Mathcad 6.0 plus, 1997.

[14] Cenk Demir, Abhyudai Singh Prediction and control of projectile impact point using approximate statistical moments. Systems and Control, 2017.

[15] Burko Lior M., Price Richard H. Ballistic trajectory: parabola, ellipse, or what? American Journal of Physics, Vol. 73, 2005, p. 516. 